\title{
Formación de formadores de lenguas extranjeras en educación intercultural por medio de una comunidad de práctica*
}

Primer semestre de 2022 - pp. 199-220 Segunda época

N.

\section{Foreign Language \\ Teacher Educators \\ Professional \\ Development on \\ Intercultural Education \\ through a Community \\ of Practice \begin{abstract}
de línguas estrangeiras em educação intercultural por meio de uma comunidade de prática
\end{abstract} \\ Formação de formadores}

Arismendi-Gómez, F. A. (2022). Formación de formadores de lenguas extranjeras en educación intercultural por medio de una comunidad de práctica. Folios, (55). https://doi.org/10.17227/folios.55-12893

* Este artículo se deriva de la investigación “Dynamiser la formation à l'éducation interculturelle des formateurs d'enseignants de langues étrangères en Colombie : une recherche-action à l'Université d'Antioquia" que llevé a cabo en el marco de mis estudios doctorales en el Laboratorio Lidilem de la Université Grenoble Alpes entre el 2016 y el 2019.

** Doctor en Ciencias del Lenguaje, Université Grenoble Alpes, Francia. Profesor asociado, Escuela de Idiomas Universidad de Antioquia.

Correo: fabio.arismendi@udea.edu.co 


\title{
Resumen
}

Este artículo de investigación presenta algunos hallazgos de un estudio cuyo objetivo fue analizar el impacto de una comunidad de práctica, como estrategia de formación de profesores universitarios de futuros docentes de lenguas extranjeras en educación intercultural. Este estudio cualitativo siguió el ciclo de la investigación-acción. Luego de un análisis de necesidades, se propuso la creación e implementación de una comunidad de práctica de la cual participaron activamente siete profesores de inglés y de francés, a lo largo de siete meses. La recolección de datos se hizo por medio de fuentes diversas, como textos narrativos escritos por los participantes, entrevistas individuales, un grupo focal y el diario del investigador. Los hallazgos plantean que las discusiones dentro de esta comunidad de práctica contribuyeron a cambios en las representaciones de los participantes sobre el concepto de interculturalidad, así como a una comprensión más profunda de las implicaciones de la educación intercultural en la enseñanza de lenguas extranjeras. Asimismo, los análisis revelan los aciertos de las comunidades de práctica como estrategia de formación docente en el tema de la interculturalidad en el medio universitario colombiano.

\section{Palabras clave}

educación intercultural; comunidades de práctica; formadores

de docentes; enseñanza de lenguas extranjeras

\begin{abstract}
This research article presents the findings of a study whose main objective was to analyze the impact of a community of practice of foreign language teacher educators as a professional development strategy addressing intercultural education. This qualitative study followed an action-research cycle. After a needs analysis, a community of practice was created and implemented in which seven language teacher educators participated actively for seven months. Data collection includes diverse sources, such as participant's narratives, individual interviews, a focus group, and the researcher's journal. Findings suggest that discussions in this community contributed to changes in participants' representations of the concept of interculturality, as well as to a deeper understanding of the implications of intercultural education on language teaching. Likewise, the analysis reveals the advantages of communities of practice as a professional development strategy focusing on interculturality at a university level in Colombia.
\end{abstract}

\section{Keywords}

intercultural education; communities of practice; teacher educators; foreign language teaching

\section{Resumo}

Este artigo de pesquisa apresenta alguns resultados de um estudo cujo objetivo foi analisar o impacto de uma comunidade de prática, como estratégia de formação de professores universitários, de futuros docentes de línguas estrangeiras em educação intercultural. Este estudo qualitativo seguiu o ciclo da pesquisa-ação. A partir de uma análise de necessidades, propôs-se a criação e implementação de uma comunidade de prática, da qual participaram ativamente sete professores de inglês e de francês, ao longo de sete meses. A coleta de dados foi feita por meio de fontes diversas, como textos narrativos escritos pelos participantes, entrevistas individuais, um grupo focal e um diário com registros do pesquisador. 0 s resultados colocam que as discussões dentro dessa comunidade de prática contribuíram para mudanças nas representações dos participantes sobre o conceito de interculturalidade, assim como possibilitou uma compreensão mais profunda das implicações da educação intercultural no ensino de línguas estrangeiras. Além disso, as análises revelam os acertos das comunidades de prática como estratégia de formação docente no tema da interculturalidade no meio universitário colombiano.

\section{Palavras chave}

educação intercultural; comunidades de prática; formadores de docentes; ensino de línguas 


\section{Introducción}

Las dinámicas del mundo contemporáneo, caracterizado por la movilidad y los intercambios cada vez más frecuentes entre personas de orígenes muy diversos, pero también marcado por un incremento del etnocentrismo y de actitudes de odio y violencia hacia ciertos grupos, han creado la necesidad de formar a los docentes en temas relacionados con la diversidad lingüística y cultural, y la alteridad. Es por esta razón que los enfoques interculturales han ganado gran relevancia en el campo de la enseñanza de lenguas extranjeras en las últimas décadas. Simon (2015) argumenta que los enfoques interculturales, aunque diversos y variados, tienen algunos objetivos en común, tales como: "Buscar una apertura a la diversidad lingüística y cultural, propiciar un trabajo de concientización y reflexión sobre la alteridad, una descentración, desarrollar actitudes de apertura, aceptación, tolerancia, empatía y capacidades de mediación en la interpretación de las diferencias" (p. 64, traducción propia). Indiscutiblemente, estos objetivos adquieren una importancia vital en el contexto colombiano actual, en el cual se busca sobreponerse a una larga historia de violencia interna. ¿Cómo contribuir desde el campo de las lenguas extranjeras a un mejor vivir en un país afectado por un conflicto interno de más de cincuenta años?
El programa de Licenciatura en Lenguas Extranjeras de la Universidad de Antioquia consideró, durante la reforma curricular que vio la luz en el 2018, que la educación intercultural y las pedagogías críticas podrían contribuir a formar un maestro no solamente más crítico y consciente de las realidades del contexto, sino también más respetuoso del Otro y cualificado para implementar acciones conducentes a un mejor vivir, el cual sería un aporte a una cultura de la paz.

Uno de los retos que esta reforma curricular conlleva es precisamente la formación continua de los profesores - formadores - de los futuros maestros en estos enfoques. Por ende, el programa ha buscado diversas estrategias de formación en diferentes temas para favorecer la apropiación de estas orientaciones por parte del cuerpo docente $y$, por consiguiente, impactar la formación de los futuros maestros. El presente estudio surge precisamente como respuesta a esta necesidad de desarrollo docente en el área de la interculturalidad en las lenguas extranjeras. Así pues, el objetivo principal de este estudio fue explorar de qué manera podría llevarse a cabo una formación en educación intercultural para un grupo de profesores - formadores de formadores- de lenguas extranjeras a nivel universitario y qué impacto tendría dicha formación. 
Siguiendo un enfoque cualitativo de investigación-acción, identifiqué en una primera etapa las necesidades de formación y las modalidades de trabajo que serían pertinentes en este contexto. Este análisis develó que la intervención debería llevarse a cabo por medio de una comunidad de práctica (CP), como estrategia de formación, en la cual los profesores pudieran intercambiar conocimientos, experiencias y prácticas, y aprender sobre la educación intercultural. Para empezar este artículo, explicaré lo que entendemos en este trabajo por interculturalidad y desarrollaré algunos elementos que sustentan la formación a través de comunidades de práctica. En segunda instancia, plantearé algunas consideraciones metodológicas de la investigación. Para terminar, presentaré algunos hallazgos que atañen directamente el impacto de la comunidad de práctica sobre los participantes.

\section{La educación intercultural en la enseñanza de lenguas extranjeras}

La educación intercultural emergió en los entornos educativos europeos de los años setenta, vinculada a la cuestión de la integración de los migrantes. El modelo intercultural se corresponde con un deseo por parte de estas sociedades de favorecer los intercambios y respetar las diferencias culturales en un contexto plural y demócrata (Demorgon, 2015; Erfurt, 2016). Según Coste (2016), las diversas situaciones de contacto entre la población de un país y los grupos minoritarios generaron preocupación sobre el asunto intercultural en el ámbito educativo. Los educadores empezaron a tomar conciencia, desde ese entonces, del papel que los sistemas educativos debían ocupar en la valoración de la pluralidad lingüística y cultural "con el objetivo de contribuir a la inclusión y a la cohesión sociales en contextos que no las favorecen de manera espontánea" (Coste, 2016, p. 68, traducción propia).

Rápidamente, la educación intercultural tuvo eco en diferentes disciplinas educativas. En el campo de la didáctica de las lenguas extranjeras, el concepto de interculturalidad comenzó a emplearse después del surgimiento de los enfoques comunicativos
(Barthélémy, 2016; Puren, 2002). Sin embargo, solo a partir de los años noventa el término se instaló definitivamente en el campo, gracias a trabajos como los de Byram (1997), quien acuñó el término competencia comunicativa intercultural y presentó su famoso modelo de los cinco saberes (savoir, savoirêtre, savoir-apprendre/faire, savoir comprendre et savoir s'engager). En el medio francófono, trabajos como los de Porcher (1986), Abdallah-Pretceille (1996) y Zarate (1993) contribuyeron al arraigo del término en esta disciplina. Más tarde, el Marco Común Europeo de Referencia para las Lenguas (Conseil de l'Europe, 2001) propuso el concepto de consciencia intercultural, que se logra gracias "al conocimiento, la percepción y la comprensión de la relación entre el 'mundo de origen' y el 'mundo de la comunidad objeto de estudio' (similitudes y diferencias distintivas)" (p. 101). Según los autores del Marco, esta noción incluye la conciencia sobre la diversidad regional y social característica de ambos mundos. Aun así, solo después de la publicación del volumen complementario (Conseil de l'Europe, 2018) del mismo documento se le asignó un lugar importante a todo lo relacionado con la interculturalidad, la mediación y la competencia plurilingüe. Dicho sea de paso, la perspectiva de interculturalidad difiere en muchos de estos trabajos o ha evolucionado con el tiempo. De hecho, en muchos de ellos se observa un deseo de acercarse a las culturas de la(s) lengua(s) extranjera(s) objeto de estudio y desarrollar competencias para entrar en contacto con sus hablantes. No obstante, algunos trabajos apuntalan la importancia de adoptar una visión amplia de interculturalidad en la clase de lenguas, refiriéndose a las diversas variedades de alteridad, tal como lo explican Blanchet y Coste (2010): "la interculturalidad recubre las modalidades y los efectos concretos de los encuentros interindividuales de y en la alteridad, alteridad irreductible en su totalidad debido a la pluralidad infinita de los fenómenos humanos y sociales" (p. 11, traducción propia).

En Colombia, como lo declara Álvarez (2014), se pasó rápidamente de una visión cultural a una visión intercultural en la enseñanza de lenguas, 
tratando de incorporar los avances en la disciplina a nivel mundial. En los medios universitarios, no han dejado de aparecer estudios que buscan incorporar una perspectiva intercultural a la enseñanza de lenguas y a la formación de maestros de esta disciplina. Así las cosas, algunos trabajos han abogado por la integración de la competencia intercultural en la clase de lenguas (Serna, 2016); a través del estudio de las representaciones de las culturas (Cruz, 2007; Hernández y Samacá, 2006); por medio de la literatura (Gómez, 2013), en las situaciones de movilidad (Viafara y Ariza, 2015); o a partir del estudio de las representaciones, los discursos o las experiencias de los futuros maestros de lenguas con la interculturalidad (Agudelo, 2007; Álvarez y Bonilla, 2009; Arismendi, 2014, 2016; Bacca, 2014; Delgadillo, 2016; Olaya y Gómez, 2013; Soler, 2016). Todos estos trabajos revelan impactos muy positivos en la toma de conciencia de los estudiantes sobre asuntos relacionados con la diversidad lingüística y cultural, la conciencia de la relación con el Otro, así como el desarrollo de actitudes de descentración y de reflexión crítica; los autores abogan por proseguir con este tipo de estudios en el contexto universitario colombiano.

\section{Una interculturalidad renovada y crítica}

Para esta investigación, adopto una "visión renovada" de la educación intercultural, retomando las propuestas de Fred Dervin, así como una visión crítica, propia del discurso latinoamericano. Con respecto a la primera, Dervin $(2011,2016 \mathrm{a}, 2016 \mathrm{~b}$, 2017) critica el enfoque reduccionista empleado generalmente en la didáctica de las lenguas. A juicio de este autor, dicho enfoque ha contribuido a presentar una imagen fija del Otro, reduciendo las identidades de los hablantes de la lengua objeto de estudio a "criterios sólidos" para caracterizarlos. Esta forma de abordar lo intercultural ha contribuido a la propagación de estereotipos sobre ciertos grupos. Por tanto, empleando la metáfora de la modernidad líquida perteneciente al pensamiento de Zygmunt Bauman, Dervin aboga por una visión líquida de la interculturalidad en educación. Esto quiere decir que, en lugar de concentrarnos en las diferencias entre los grupos y de representar al Otro como alguien que ostenta todas las características del grupo mayoritario al que pertenece, deberíamos enfocarnos en "el estudio de la negociación de la construcción conjunta de las diversas diversidades de los individuos en presencia" en un intercambio (Dervin, 2011, p. 12, traducción propia). Más llanamente, esto significa prepararnos en la clase de lengua para el encuentro del Otro, como un sujeto singular.

Por otro lado, el discurso latinoamericano ha introducido una visión crítica de la interculturalidad que reivindica las luchas de las comunidades indígenas y afro en la defensa de sus derechos en todas las esferas de la sociedad. De este modo, esta visión "se entiende como proceso, proyecto y estrategia que intenta construir relaciones - de saber, ser, poder y de la vida misma - radicalmente distintas" (Walsh, 2010 , p. 91). Se busca entonces realizar transformaciones profundas en la sociedad (Ferrão, 2010) empoderando a los grupos y a las personas que han sido históricamente excluidas o consideradas como inferiores. Todo esto se realiza desde una perspectiva decolonial, enfoque que empieza a encontrar su lugar en la enseñanza de lenguas extranjeras en el continente. Así por ejemplo, el trabajo de GranadosBeltrán (2016) constituye un aporte a la didáctica de las lenguas, al propender por la integración de una perspectiva intercultural decolonial en la formación de futuros maestros de lenguas. En palabras de este autor, el objetivo de esta perspectiva es "cuestionar, desplazar y subvertir conceptos y prácticas dejadas por la herencia colonial con el objetivo de intervenir, construir, crear y liberar a través de una práctica decolonial" (p. 181, traducción propia). En el plano teórico, este trabajo constituye un entramado de estas perspectivas puesto que, a pesar de ser originarias de contextos alejados y diferentes, se encuentran aspectos en común. Así, tenemos entre los principios fundantes la idea de buscar un conocimiento más profundo del Otro y de darle su lugar, comprendiendo su pluralidad y sus identidades. Se busca superar el estadio del reconocimiento o de la mera tolerancia, con miras a la construcción con- 
junta de una sociedad más equitativa que propicie un mejor vivir.

\section{Formación de maestros de lenguas en educación intercultural}

Tal como se presentó en la Introducción, se hace necesario implementar estrategias de formación permanente del profesorado en ejercicio, que permitan aprehender las implicaciones de la educación intercultural en la enseñanza de lenguas. Los investigadores coinciden en la importancia de la formación en grupo, del trabajo reflexivo, de la exploración de las creencias y representaciones de los profesores sobre el tema y del acercamiento teórico. Verbigracia, Aguado et al. (2008) postulan que, aunque los conocimientos (savoirs) y las habilidades (savoir-faire) son esenciales, la formación en educación intercultural debe enfocarse principalmente en el savoir-être, es decir, en las actitudes de los maestros. Por ende, las autoras proponen iniciar los procesos de formación analizando las creencias y prácticas de los maestros con respecto a la educación intercultural; el enfoque no debe limitarse a aprender técnicas y metodologías. Adicionalmente, sostienen que la formación debe realizarse en los lugares de trabajo de los maestros y por medio de comunidades de práctica.

Otras estrategias de formación han comprendido la participación de profesores de lenguas en plataformas de trabajo colaborativo donde pueden intercambiar conocimientos y experiencias con personas de otros países y reflexionar sobre la dimensión plurilingüe e intercultural de la enseñanza de lenguas (Bastos, 2015). Desde la posición de Bastos (2015), los programas de formación en educación intercultural deben partir de las necesidades de los maestros y deben integrar las dimensiones curricular, interpersonal, política y social.

Análogamente, otra gran línea de trabajos de la formación de maestros de lenguas en educación intercultural ha surgido en los medios francófonos, por medio del uso de elementos biográficos en la formación (Galligani, 2014; Molinié, 2019; Perregaux, 2002; Simon, 2014; Thamin y Simon,
2009). Particularmente, se hace referencia al uso de biografías lingüísticas (Molinié, 2011; Thamin y Simon, 2009) o de herramientas de tipo portafolio, como la "autobiografía de encuentros interculturales” (Byram et al., 2009) creada por el Consejo de Europa. Este tipo de trabajos ha demostrado que la escritura de textos sobre lo plurilingüe y lo intercultural en la propia historia de vida favorece la reflexión y la concientización sobre estos temas, lo cual impacta a la postre las prácticas de enseñanza (Simon, 2015).

Como se verá, estas recomendaciones de la literatura inspiraron la propuesta de formación que se implementó en la presente investigación. Es menester señalar que la mayoría de publicaciones que abordan este tema en la formación de maestros en Colombia lo hacen en el marco de la formación inicial (Agudelo, 2007; Arismendi, 2016; GranadosBeltrán, 2016; Peña et al., 2019) o continuada de docentes en ejercicio (Gamboa Diaz, 2019; Gamboa Diaz et al., 2019; Serna et al., 2016). En el desarrollo de la presente investigación no se encontraron publicaciones sobre la formación permanente en educación intercultural de los formadores de maestros de lenguas en los medios universitarios en Colombia, de modo que este tipo de estudios contribuirá a la reflexión sobre los procesos de formación intercultural continuada.

\section{Las comunidades de práctica como estrategia de formación}

El término comunidades de práctica (СP) se debe originalmente a Étienne Wenger (1998), quien lo acuñó en el mundo de las organizaciones. Este autor enfatiza en el aprendizaje colaborativo que se logra cuando un grupo de personas que comparten un interés crean una empresa conjunta para perseguir un objetivo común. A su vez, Tremblay (2005) define de manera sucinta esta estrategia de formación:

Una comunidad de práctica es generalmente definida como un grupo de personas que tienen un campo de experticia o una práctica profesional en común, y que se encuentran para intercambiar, compartir y aprender los unos de los otros, bien 
sea de manera presencial o virtual. (p. 54, traducción propia)

Como lo hace notar Wenger (1998), tres dimensiones garantizan el funcionamiento de las CP: el compromiso mutuo, la empresa conjunta y el repertorio compartido.

Por su parte, Bozu e Imbernon (2009) indican el potencial de las CP en la formación continua del profesorado universitario, sobre todo porque contribuyen a sobrepasar las situaciones de aislamiento profesional, características del medio universitario. Asimismo, Tremblay (2006) sostiene que las CP pueden ser informales, apoyadas por la organización o estructuradas de manera más formal. Para Riel y Polin (2004, citados por Bedoya et al., 2018), las CP se pueden clasificar según sus objetivos: centradas en la realización de una tarea, en la mejora de una práctica o en la producción de conocimiento. En el campo de las lenguas extranjeras, la noción de CP resulta interesante para los procesos de formación continuada, puesto que se genera un espacio para construir conocimiento localizado y aprender los unos de los otros, no únicamente para consumir el conocimiento elaborado en otros lugares. En este sentido, este modelo de formación puede caracterizarse como colaborativo, crítico y práctico (Cárdenas-Beltrán et al., 2010) ya que los profesores son considerados como profesionales, en una perspectiva de desarrollo mas no de entrenamiento técnico.

\section{Metodología}

Este estudio siguió los principios de la investigación cualitativa, según los cuales se procura comprender los fenómenos, individuales o sociales, desde la perspectiva de los sujetos y en los contextos donde estos ocurren, recurriendo a métodos rigurosos de recolección y análisis de la información (Anadón, 2006; Blanchet y Chardenet, 2011). En vista de que este estudio procuraba no solo describir $y$ comprender el contexto, sino también contribuir a su transformación, se realizó una investigación-acción (Macaire, 2007). Esta investigación constituye entonces un primer ciclo completo de investigación-acción, que incluyó un diagnóstico, la búsqueda e implementación de estrategias de formación y su posterior análisis. La primera etapa consistió en un diagnóstico, por medio de datos provenientes de documentos oficiales, entrevistas a los administradores de la Escuela de Idiomas y del programa de Licenciatura, así como de encuestas al grupo de docentes del programa. El análisis de esta información arrojó que una CP sería la estrategia de formación más apropiada durante la intervención. Luego de su implementación, un grupo focal llevado a cabo con los participantes, así como los materiales construidos durante la CP permitieron comprender el impacto de esta estrategia de formación.

\section{Implementación de la comunidad de práctica}

Treinta y dos formadores de maestros participaron en la encuesta realizada en la primera parte del estudio. Los resultados permitieron explorar las representaciones iniciales de estos profesores sobre el tema, e identificar un grupo de alrededor de quince formadores interesados en participar en la CP, quienes fueron contactados para la etapa de intervención del estudio. Doce formadores respondieron manifestando su interés de formar parte de la CP y concurrieron en la primera parte de la recolección de datos, que incluyó la elaboración de un texto narrativo biográfico (biographie langagière) y una entrevista como complemento al texto. Este ejercicio biográfico procuraba, por una parte, conocer de manera profunda a los participantes, para los objetivos de la investigación, y, por otro lado, favorecer la reflexión sobre estos temas, antes de iniciar los intercambios en la CP.

En calidad de investigador y animador de la comunidad de práctica, convoqué a una primera reunión a los doce profesores interesados. En esta reunión presenté mi propuesta de trabajo, la cual fue acogida por los participantes, enriquecida y apropiada a lo largo de los meses que duró la CP. A este encuentro asistieron siete profesores (cuatro de francés y tres de inglés), quienes se convirtieron en los miembros activos de la CP a lo largo de su implementación, entre enero y agosto del 2018. Los otros cinco profesores manifestaron su interés en unirse 
a la CP en algún momento, pero su participación nunca se concretó debido a factores relacionados con el tiempo y la carga laboral. Durante esta primera reunión, acordamos realizar encuentros de dos horas cada quince días. Esto permitiría tener una semana sin encuentro colectivo, para realizar las tareas acordadas en la comunidad, tales como lectura de textos, reflexiones, planes de clase, etc.

El trabajo en esta CP se enfocó en tres componentes. En primera instancia, se realizó un trabajo reflexivo sobre la interculturalidad en la historia de cada profesor. Este comenzó antes de iniciar los intercambios, por medio de la redacción de la biografía lingüística y la participación en la entrevista. A lo largo de la CP, el trabajo biográfico ocupó un lugar importante puesto que la reflexión se enriqueció, al compartir y leer los textos de sus pares. En efecto, durante la cuarta sesión colectiva, y por sugerencia de una de las participantes, se acordó como tarea la lectura de los textos biográficos escritos por cada profesor. Se propuso una guía de preguntas para la discusión de dichos textos durante la quinta sesión colectiva. No obstante, en este artículo no se profundizará sobre los textos narrativos, sobre la forma en que fueron abordados ni sobre el impacto de esta estrategia en los participantes, pues estas temáticas constituyen per se el tema de otro texto completo.

En una segunda instancia, la CP favoreció la apropiación teórica sobre interculturalidad en el campo de las lenguas extranjeras, principalmente desde algunas perspectivas europeas y latinoamericanas. Finalmente, hubo un componente práctico, en el que los profesores planearon algunas sesiones de clase tomando en cuenta las reflexiones realizadas en la CP. Estos planes de clase fueron discutidos, reelaborados e implementados por tres de las profesoras participantes, en agosto del 2018.

El trabajo efectivo de la CP duró 23 semanas, durante las cuales se alternaron reuniones de todo el grupo (cada quince días), trabajo individual y algunas sesiones de trabajo por lengua — profesores de inglés y de francés de forma separada- para la preparación de los planes de clase. En cuanto al primer tipo, se realizaron doce sesiones colectivas con todos los profesores. Todos los participantes leían todos los textos acordados (véase el anexo 1) y durante la sesión se discutía sobre la lectura o material estudiado previamente. Como apoyo a la discusión, el investigador y líder de la comunidad aportaba algunas preguntas generadoras y, en algunas oportunidades, presentaba material complementario o audiovisual. Adicionalmente, se creó un espacio virtual para la CP en la herramienta Google Classroom. Allí se hacían anuncios, se publicaba el material de trabajo acordado y cada participante podía compartir materiales y comentarios. Sin embargo, esta herramienta fue subutilizada. Para llevar un registro del trabajo, todas las sesiones colectivas fueron grabadas en audio y se realizaron protocolos de cada sesión que sintetizaban los puntos esenciales de los textos y de la discusión, además de las tareas por desarrollar. Todos los participantes tenían acceso a dichos textos antes del siguiente encuentro. Con respecto a las tareas, estas se acordaban entre todo el grupo. De hecho, el investigador hizo algunas propuestas de lecturas al inicio, pero los participantes sugirieron nuevos textos y actividades a medida que avanzaba el trabajo.

\section{Participantes}

El grupo de participantes de la CP estuvo constituido por siete profesores. ${ }^{1}$ Se trata de seis mujeres y un hombre, cuyas edades se encuentran en rangos bastante amplios (entre menos de treinta y más de sesenta años). Cuatro personas enseñan francés y las otras tres, inglés. En el momento en que se adelantó la investigación, cinco personas eran profesores de tiempo completo (dos de planta y tres ocasionales), y dos tenían contratos de cátedra. Todos los participantes de la CP poseen el grado de magíster y habían vivido en el extranjero, en países anglófonos o francófonos, en algún momento de sus vidas. Todos poseen experiencias docentes muy variadas, y en algunos casos extensas, que incluyen la enseñanza en instituciones educativas públicas o

1 El investigador, y autor del artículo, también formó parte del estudio, como octavo participante. Sin embargo, con el fin de tomar distancia, solo se presentan los hallazgos reportados por los otros siete profesores. 
privadas, centros de lenguas y en el nivel universitario, en Colombia. Algunos han tenido experiencia docente en el extranjero, por ejemplo, por medio de la asistencia de lenguas.

\section{Recolección y análisis de datos}

Además de los datos mencionados, durante la implementación de la CP y su posterior análisis, se recurrió a las siguientes fuentes de datos: el diario de campo del investigador; un grupo focal, realizado después de haber finalizado la CP y que buscaba evaluar el trabajo realizado y analizar el impacto de la CP en los participantes (véase la guía en el anexo 2); y todos los documentos producidos durante la implementación de la CP (planes de clase y observación de su implementación).

Los datos recolectados se analizaron siguiendo la técnica del análisis de contenido (Bardin, 2013; Burns, 1999), con el apoyo del programa Nvivo 10. Este tipo de análisis busca la descripción sistemática del contenido de comunicación con miras a interpretarlo, a partir de la inferencia (Bardin, 2013). Después de haber transcrito todos los datos, se procedió a su codificación, recurriendo a categorías previas y emergentes. Con el fin de garantizar un análisis riguroso, se recurrió a la triangulación de los datos (Burns, 1999). En efecto, como ya se señaló, la información provenía de fuentes diversas y fue recolectada en diferentes momentos de la investigación. Asimismo, después de realizar los análisis, los participantes recibieron los borradores de dos capítulos de la tesis doctoral, que presentaban los hallazgos con el fin de validar los análisis. En esta línea es importante aclarar que todos ellos firmaron un formato de consentimiento para participar en este estudio. Su identidad fue protegida durante la investigación y la divulgación de los hallazgos.

\section{Hallazgos}

El análisis de los datos permitió observar transformaciones en el discurso de los profesores con respecto a la educación intercultural en la enseñanza de lenguas. Este discurso revela una comprensión más profunda de lo intercultural en este campo.
Asimismo, los datos permiten entender de qué manera la CP desempeñó un papel importante en su formación permanente.

\section{Evolución de las representaciones sobre la interculturalidad}

En todos los participantes se logra apreciar una evolución de sus representaciones con respecto al concepto de interculturalidad. Si bien desde la primera entrevista, realizada en enero del 2018, se observaba una conciencia y un interés sobre este tema - que los llevó a integrar este colectivo- los datos obtenidos en el grupo focal, realizado a finales de julio del mismo año, muestran que el trabajo dentro de la CP influyó sobre su comprensión y apropiación del concepto, tal como lo revela el siguiente fragmento:

uno tenía una idea diferente de interculturalidad que era /solamente como con otras naciones otras culturas extranjeras no aquí mismo / eh la interculturalidad a nivel de de ciudad o a nivel de región/ que es el encuentro con el otro esa noción de/ que es el encuentro con el otro / que es diferente eh eso me llevo yo porque me sonó pues mucho mucho y no lo tenía tan claro cuando / cuando lo inicié. (Patricia, profesora de francés, grupo focal)

En este fragmento se observa una complejización del concepto. Mientras que antes entendía la interculturalidad desde una visión de intercambio binario entre personas originarias de dos países diferentes (por ejemplo, Colombia y Francia), el trabajo en CP le permitió entender otras visiones más amplias de la interculturalidad que propugnan las relaciones alteritarias. Esto nos lleva a los postulados de Blanchet y Coste (2010) y de Dervin $(2011,2016 a$ y 2016b, 2017), citados en el marco teórico. En la misma línea, otra profesora apunta una evolución en su forma de entender el concepto, particularmente al pasar de una visión inicial muy "instrumental" a una comprensión más profunda:

2 Convenciones de transcripción: / marca una pausa corta, // marca una pausa un poco más larga. Se utilizan seudónimos para proteger la identidad de los participantes. 
para mí fue un cambio / total / [...] haber estado aquí con ustedes y hacer las lecturas eh la enriqueció totalmente $[. .$.$] o sea cambió totalmente$ esa visión / lo que yo pienso que es un reto para mí personalmente ahora es llevar todo ese cambio y esa nueva visión al aula de clase / ¿cierto? / eh pero para mí / sí es o sea / la visión de cultura e interculturalidad con que yo inicié al comienzo / que que era una netamente instrumental sí cambió totalmente. (Margarita, profesora de francés, grupo focal)

Otros participantes se refieren a la idea de dinamismo, lo que nos muestra el paso de una visión estática del concepto hacia una visión más dinámica:

es ese dinamismo esa idea de movilidad / de / de cosas que se están moviendo que me dejó pues / otra vez no solo la lectura sino el trabajo de de pensar en lo intercultural como lo dice el autor este Dervin más en lo inter que lo cultural / es decir esa idea de que es que hay movimiento pues permanente / en los seres. (Estella, profesora de francés, grupo focal)

Los tres fragmentos citados reflejan, entonces, evoluciones en la forma de comprender la interculturalidad. No se trata únicamente de apreciaciones en el discurso de los profesores, puesto que el trabajo de planeación de cursos realizado dentro de la CP permitió concretar estas ideas en la clase de lenguas. Además, las apreciaciones de estas tres profesoras nos permiten también reflexionar sobre la evolución epistemológica de la didáctica de las lenguas y culturas, en lo que concierne a la cultura y la interculturalidad (Defays, 2018; Puren, 2002, 2011). Según Defays (2018), en la enseñanza de lenguas se pasó de un énfasis en los saberes sobre las culturas extranjeras o cultura meta -enfoque civilizacional-, a un enfoque sobre las habilidades para entrar en contacto con otras culturas, perspectiva predominante en los enfoques comunicativos. Posteriormente, de acuerdo con el mismo autor, pasamos a un enfoque basado en las actitudes y en la búsqueda de un mejor vivir, tendencia predominante en la actualidad y objetivo principal de los enfoques interculturales. Esta evolución está claramente esbozada en el segundo testimonio.
En efecto, Margarita manifestó, en diversos momentos durante los intercambios en la $\mathrm{CP}$, que en su formación como profesora de francés se enfatizó en el enfoque civilizacional. Por esta razón, arguye que su visión "cambió totalmente" gracias a las lecturas $\mathrm{y}$ a los intercambios en la CP.

Para todos los participantes, la CP tuvo un rol importante en la ampliación de su comprensión de la interculturalidad. Los datos permiten observar que uno de los aspectos más impactantes es la interculturalidad entendida como la relación con el Otro, de manera amplia. Esto nos remite a Dervin, para quien "este paradigma asume que cada individuo es diferente (y no solamente el Otro/el extranjero en los encuentros interculturales) y similar al mismo tiempo" (Dervin, 2017, p. 60, traducción propia). Siguiendo los postulados de este autor, es posible afirmar que la CP facilitó este intercambio con el Otro. Aunque no profundizaremos en este tema, este encuentro consciente con el Otro se logró, en parte, gracias al trabajo biográfico. La redacción individual, así como la posterior lectura y discusión de los textos narrativos escritos por los colegas, sirvió como punto de entrada para poner sobre la mesa, recurriendo a la fórmula del mismo autor, las diversas diversidades de los individuos que formaban parte de la CP. El énfasis se dio así en los procesos de co-construcción y negociación entre los individuos presentes.

\section{Comprensión más profunda e informada de la educación intercultural en la enseñanza de lenguas}

Los datos sugieren que el trabajo de la CP favoreció las comprensiones y prácticas de enseñanza de los profesores participantes con respecto al tema de la interculturalidad. Por un lado, las discusiones revelan conexiones entre las teorías estudiadas y la historia personal de cada uno. Por otro lado, las discusiones muestran que es necesario generar conexiones entre diferentes perspectivas teóricas sobre la interculturalidad en la enseñanza de lenguas en nuestro contexto. Finalmente, se observan algunos efectos de la CP en el quehacer docente, desde el punto de vista reflexivo y de la planeación. 
En primer término, las discusiones en la CP posibilitaron conexiones entre las teorías estudiadas, la propia historia de vida, y la reflexión sobre el quehacer como docentes de lenguas. Así lo deja entrever la siguiente aserción, extraída de una discusión durante un encuentro de la CP, en la que uno de los profesores manifiesta su sentir con respecto a la interculturalidad:

algo que a mí me pasó con las experiencias después de las lecturas / las lecturas de cierta manera me han ayudado como a sentir / ese tema de la interculturalidad//como algo más cercano / ya no es como viéndolo como una teoría más / yo antes lo veía como una teoría más que me tocaba de cierta manera manejar por ser profe de lenguas sino que ya estoy empezando a sentir como algo más cercano / y eso lo asocié como con lo de las experiencias que escribimos que compartimos la última vez que nos vimos / porque uno se da cuenta / si uno hace como una especie de de// flashback/uno se da cuenta [sic]// que uno tuvo /muchas experiencias de tipo intercultural y que no sabía. (Felipe, profesor de francés, transcripción de la discusión llevada a cabo el 23 de abril del 2018)

Más allá de una simple comprensión del término, lo que este fragmento refleja es la concientización que Felipe logró gracias al trabajo realizado, donde se conjugaron lecturas, trabajo reflexivo sobre su propia historia de vida e intercambio con otros colegas. Así pues, su comentario evidencia la trascendencia de los tres componentes de formación de la CP mencionados en la metodología. Asimismo, se constata que la formación en educación intercultural no puede hacerse únicamente por medio de un curso magistral o a partir de constructos teóricos. Al contrario, dicha formación exige reflexionar sobre sí mismo, sobre su propia pluralidad y su relación con el Otro (Galligani, 2014; Simon, 2014). Al respecto, Porcher y Abdallah-Pretceille (1998) postulan que

La diversidad es constitutiva de la naturaleza del hombre y el reconocimiento de su propia diversidad es una de las condiciones para poder reconocer la diversidad del Otro. Quien es incapaz de ver la multiplicidad de su ser y su riqueza interior no puede tener acceso a la riqueza del Otro. Por lo tanto, cualquier trabajo de formación implica también un trabajo sobre sí mismo, en particular a nivel de las referencias y elecciones éticas, y no únicamente sobre el Otro. (p. 93, traducción propia)

Discurrir sobre estos asuntos no es tarea fácil, por ende, debe hacerse de forma voluntaria y es en esta medida que las CP resultan eficaces para este tipo de trabajo. Una formación en interculturalidad supone que el sujeto se sienta concernido. Así lo expone, a manera de conclusión durante el grupo focal, otra de las profesoras:

escribir esa autobiografía // eh // y pero después compartirla acá y hablar de las autobiografías de los otros compañeros / um me sirvió mucho para para / para fortalecer ese principio de que esto tiene que pasar por mí mismo que un trabajo desde la interculturalidad eh me indica es ponerme yo ahí como persona y también pensar en mi historia. (Paola, profesora de inglés, grupo focal)

En segundo término, las discusiones en la CP permitieron analizar, problematizar e incluso deconstruir diversas perspectivas teóricas sobre interculturalidad en la enseñanza de lenguas. En el siguiente fragmento, extraído de la conversación llevada a cabo en una de las sesiones de la CP y luego de haber estudiado en detalle el modelo de Byram, una de las profesoras manifiesta sus reservas sobre la utilización de dicho modelo en el contexto colombiano:

veo problemático utilizar compar [los demás ayudan a completar la palabra] compartimentos para poner algo que es tan complejo y donde muchas cosas confluyen y cómo se ve cada cosita en el salón de clase aún más / porque cuando hacemos algo en el salón de clase puede que estemos trabajando en diferentes frentes / [...] la segunda cosa que quería contar / es que igual es una visión muy europea de esto de la interculturalidad / o sea hay una posición de un autor desde un punto geográfico y todo lo que eso significa y que yo y que yo desde aquí pienso como bueno / sí me puedo nutrir de muchas cosas pero me falta me 
queda faltando mucho que toque más con lo / con nuestras realidades para ese trabajo intercultural. (Paola, profesora de inglés, transcripción de la discusión llevada a cabo el 11 de febrero del 2018)

Emergen entonces dos elementos importantes de la declaración anterior. Por un lado, la dificultad percibida por esta profesora de segmentar la competencia intercultural en diferentes componentes. Y, por otro lado, el carácter eurocentrista del modelo de Byram para la interculturalidad en la enseñanza de lenguas. Aunque dicho modelo, así como los trabajos del Consejo Europeo, predominan en muchos trabajos en el área, resulta imperioso buscar otras perspectivas - o establecer puentes con otras miradas- que puedan responder más claramente a las particularidades sociales e históricas del continente latinoamericano.

Es por estas razones que nuestra apuesta en este trabajo es por construir puentes entre una mirada renovada y crítica de la interculturalidad. La primera, aunque también de origen europeo, nos hace pensar en el encuentro con el Otro, de manera amplia y apartándonos de criterios esencialistas, como hemos insistido a lo largo de este trabajo. La segunda nos remite al empoderamiento de grupos y personas históricamente excluidas. La interculturalidad crítica resulta así relevante para la intervención en la clase de lenguas, en la cual se puede, por ejemplo, deconstruir las ideas de colonialidad presentes en los materiales de aprendizaje o en los mismos discursos que circulan en el aula. Esta mirada, que aboga por una perspectiva decolonial, estuvo presente en las discusiones en la CP, como lo señala otra de las participantes:

hubo algo que me resonó mucho y es el / em / cuando estábamos hablando de poscolonialidad y colonialidad / eso es un asunto muy importante que / que yo creo que /// es una oportunidad como para nosotros eh ubicarnos en este asunto de las lenguas extranjeras en el contexto nuestro ampliado de Colombia y Latinoamérica / que me parece que es importante que nosotros nos veamos en relación a [sic] nosotros mismos no a / no es descartar los otros no pero sí entrar como en un diálogo pero como en un diálogo eh / en el que nosotros tengamos como más conciencia de de la historia propia. (Susana, profesora de inglés, entrevista final)

Lo que sobresale, por ende, de las discusiones y del trabajo realizado en la CP es la necesidad de establecer puentes entre las diferentes visiones de la interculturalidad y de construir conocimiento contextualizado sobre este tema para el área de lenguas. Así lo explicita una de las participantes durante el grupo focal:

yo sí soy de la posición de que los profesores de lengua extranjera nos hemos parado más desde el otro que desde nosotros mismos y he pasado / es o sea eso también se dio a [...] en mi propia historia $[\ldots]$ / he ido como cambiando mis mis visiones pero [...] lo que yo veo de nuestro campo es que / estamos muy estamos muy permeados por el conocimiento y las culturas angloparlantes [...] yo sí pienso que tenemos una deuda muy grande en nuestro campo / de valoración de lo propio / de valoración de lo nuestro desde la / desde la construcción de conocimiento. (Paola, profesora de inglés, grupo focal)

Para terminar, las reflexiones lograron trascender el espacio de la CP y reflejarse en el quehacer de los docentes. Por ejemplo, en la siguiente declaración, se explicita cómo la CP permitió ganar conciencia sobre una acción específica para implementar en el aula:

yo algo que volví más consciente desde las conversaciones que tuvimos es / involucrar un poco más los estudiantes como tal / porque yo creo que tengo como esa conciencia de bueno sí hablemos como de Colombia y como en su riqueza pero a veces no exploto como tal la riqueza que ya tengo en el aula de los estudiantes que vienen de otras regiones / y que probablemente tienen un poquito más de de / o que pasan por situaciones para acomodarse a este contexto y que quiero que se sientan valorados desde lo que traen a esta a este espacio digamos predominantemente paisa ¿cierto? /. (Camila, profesora de inglés, grupo focal)

La CP se convirtió por consiguiente en un espacio para discutir sobre temas relacionados con asuntos internos a Colombia, en el contexto del 
posconflicto, a través de preguntas que se inscriben en una dinámica de interculturalidad crítica (Granados-Beltrán, 2016; Walsh, 2010). Así por ejemplo: ¿Cómo favorecer un mejor vivir desde la clase de lenguas? ¿Qué puede hacer el maestro de lenguas desde su quehacer en la situación del posconflicto? ¿Cómo favorecer, en la clase de lenguas, el conocimiento y la valoración de las comunidades excluidas históricamente en el país?

Todas estas discusiones se concretaron en el trabajo práctico de creación e implementación de planes de clase. En efecto, dentro de la CP se creó una unidad para un curso de inglés y otra para francés, que fueron implementadas en la nueva versión del pénsum de la Licenciatura en Lenguas Extranjeras, en el segundo semestre del 2018. A manera de ejemplo, observemos lo que ocurrió con los profesores de francés.

Después de varios encuentros para planear el curso, el grupo decidió crear una unidad cuyo tema central fue la descripción de personas, tema que se aborda habitualmente en los primeros niveles y que aparece igualmente en la mayoría de libros de texto de primer nivel. Sin embargo, a partir de las discusiones y siguiendo los principios de la educación intercultural estudiados en la CP, los profesores buscaron, adaptaron y crearon materiales en francés que se corresponden con las particularidades del contexto. Por tanto, se partió de un descubrimiento de celebridades a nivel global, pero el trabajo detallado implicó concentrarse en personajes colombianos oriundos de comunidades excluidas y poco conocidas por los estudiantes, tales como grupos indígenas y afrocolombianos, apostando así a la generación de un espacio que permitiera ahondar en las representaciones sobre diversos grupos.

La unidad de enseñanza creada e implementada propone además el desarrollo y la integración de todas las competencias lingüísticas, así como diversas modalidades de trabajo (individual, en parejas o grupos pequeños y en gran grupo). Los profesores realizaron un gran trabajo de adaptación y didactización de materiales auténticos, ${ }^{3}$ así como de creación de nuevos materiales que, por cuestiones de espacio, no es posible presentar en este artículo. El objetivo final de dicha unidad consistía en realizar un texto corto y un video en parejas, para responder a la pregunta: ¿Cómo somos los colombianos? Las reflexiones realizadas en clase permitieron a los estudiantes concluir que no existe una única manera de responder a dicha pregunta, puesto que no es posible hacer una descripción generalizada de todos los habitantes de un país.

\section{Aciertos de la formación en comunidad de práctica}

Tres aspectos emergieron como aciertos durante la implementación de esta CP: el tipo de intercambios que se llevaron a cabo, caracterizados principalmente por su horizontalidad y por su carácter voluntario; la dimensión temporal y la dimensión práctica.

En primer lugar, los intercambios entre los colegas participantes fueron horizontales. En otras palabras, las voces de todos fueron escuchadas y ninguno de ellos adoptó la posición de experto que formaría a los otros:

la dinámica del grupo que fue también muy horizontal / o sea no / no fue como el experto que vino a enseñar de interculturalidad / sino como también nosotros fuimos construyendo / aprendiendo los unos de los otros eh en un tema de los que los estábamos leyendo / eso me parece muy importante también en una comunidad de práctica. (Paola, profesora de inglés, grupo focal)

En este sentido, uno de los aspectos más importantes para el éxito del trabajo realizado es la actitud que cada uno de los participantes adoptó:

3 Se didactizaron, por ejemplo, los siguientes videos, que sirvieron como punto de partida para el trabajo lingüístico e intercultural:

Sobre la apariencia física que permite cuestionar las nociones de belleza https://www.youtube.com/watch?reload=9\&v=BEOpMVfvDK4

Être une femme noire en Colombie, dangereux ? (0'51-3'14) https://www. youtube.com/watch?v=jWTJuxf45Ks\&t=26s 
yo pienso que / que lo que lo hizo más una comunidad de práctica fue la actitud que todos tomamos porque / porque / como dijo Patricia de que [sic] éramos como un grupo de colegas y la igualdad [...] porque yo he ido a otras partes donde sí se dice que es comunidades de práctica y los expertos son los que brillan ¿cierto? / (Margarita, profesora de francés, grupo focal)

Los formadores mostraron un compromiso mutuo con miras a lograr la empresa conjunta de formación en educación intercultural. Por ende, la dinámica de las interacciones en esta CP se ajustó así a las dimensiones propuestas por Wenger (1998): el compromiso mutuo, la empresa conjunta y el repertorio compartido.

Otro aspecto importante se relaciona con el hecho de haber participado de forma voluntaria en la CP, sin ningún tipo de presión de la administración o de necesidad de recibir un certificado de participación. Por el contrario, se trató de una propuesta de un colega que interesó a los profesores:

nos hacen falta estos espacios en la escuela / como de espacios que no sean // castigables (risas) castigables si vas si no vas / lo tiene que hacer o tiene un certificado / el no tener esa presión sino que fue algo que desde el principio se hizo de la voluntad yo creo que marca / la creación de un espacio distinto / entonces para mí este tipo de iniciativas que vienen como desde nosotros en este caso desde tu propuesta pero nosotros dijimos sí porque de alguna manera teníamos ahí unos intereses y una voluntad desde lo / desde lo personal y profesional entonces ya eso marca una / una historia distinta (Paola, profesora de inglés, grupo focal)

Es menester señalar que los participantes reconocieron la importancia del liderazgo de la $\mathrm{CP}$, en este caso del investigador, en la medida en que se necesita que alguien convoque y se encargue de algunas tareas que puedan garantizar el buen funcionamiento de la comunidad.

Un segundo acierto tiene que ver con la dimensión temporal. La formación en CP se realiza durante un periodo relativamente largo, en este caso siete meses, lo cual redunda en un mayor tiempo de asimilación de las teorías y de lo que se estudia. Esto, en comparación con los talleres o cursos de corta duración que se ofrecen más comúnmente para la formación de profesores universitarios:

un trabajo así constante y largo en el tiempo es más productivo que pequeños talleres aislados [...] un grupo de trabajo donde leemos discutimos / donde no está el experto allá arriba diciéndonos qué hacer y cómo hacer las cosas / sino que todos estamos aprendiendo desde / como validando también lo que hacemos nosotros en el en el aula de clase / desde nuestra experiencia / eh entonces por ese lado muy interesante. (Patricia, profesora de francés, grupo focal)

El último aspecto concierne a la dimensión práctica del trabajo realizado en la CP. Para los participantes, es imperativo que este tipo de formación no se quede únicamente en el plano teórico, sino que trascienda hacia la práctica:

también quiero resaltar el punto que menciona Estella y es / es eso es haber abierto el espacio para que esas teorías que que exploramos se vieran materializadas como de forma práctica / porque yo creo que a veces ese es uno de los puntos más difíciles / es aterrizar esas teorías y ver bueno yo en el aula yo cómo llevo esto eh / a cabo / entonces siento que ese espacio fue muy muy valioso para mirar qué de todo lo que exploramos podemos / desde nuestra práctica llevar al aula de clase. (Camila, profesora de inglés, grupo focal)

El trabajo teórico y reflexivo fue esencial, pero habría quedado incompleto si no se hubiera procedido a la práctica.

\section{Algunas dificultades}

La principal dificultad para el desarrollo de esta CP fue el tiempo. En efecto, las cargas de los profesores universitarios - tanto de tiempo completo como de cátedra- son elevadas, lo cual dificulta el poder comprometerse en una empresa de este tipo. De un grupo inicial de doce formadores interesados, solo siete, además del investigador, pudieron participar activamente a lo largo de los meses que duró la CP. En este sentido, este estudio encuentra conclusiones 
análogas a las presentadas por otros investigadores (Bedoya et al., 2018; Gómez et al., 2018). Las actividades más cortas de formación, como talleres y cursos de corta duración, pueden tener entonces más eco en los profesores que tienen menos tiempo disponible, aunque como lo señaló una de las participantes, su impacto puede ser menor. Adicionalmente, debido a los recursos limitados de la institución pública en la que se llevó a cabo el estudio, no se logró obtener ninguna remuneración para los profesores cátedra que participaron.

Aunado a las cargas de trabajo, las llegadas tarde a los encuentros y algunas ausencias fueron señaladas como aspectos por mejorar durante el grupo focal que se realizó al final. A pesar de que el horario fue propuesto por los mismos participantes, nunca impuesto por el investigador, este fue difícil de respetar durante todo el tiempo que duró la CP. Con respecto a la asistencia a las sesiones de encuentro programadas, se observó una disminución en el tercer y quinto mes, y luego al final. Para terminar, aunque los participantes reconocieron la importancia del trabajo práctico, insisten en que este se realice de forma permanente desde el inicio y no se deje para el final.

\section{Implicaciones para los procesos de formación continua del profesorado}

Los hallazgos de este estudio permiten identificar algunas pistas concretas de trabajo cuando se desea implementar acciones de formación en educación intercultural para profesores universitarios por medio de CP.

En primer lugar, un trabajo de formación en CP debe hacerse de manera voluntaria. Los hallazgos muestran que los resultados de esta intervención fueron positivos en gran medida porque no se trató de algo impuesto por la administración de turno. En realidad, comprometerse en una empresa de formación de este tipo, que implica un trabajo de reflexión personal y de intercambio de experiencias, no se logra fácilmente cuando es impuesto.

En segundo lugar, resulta primordial abrir un espacio que permita un acercamiento a lo biográfico durante la formación. Aunque por cuestiones de espacio no insistimos mucho en este tema en este artículo, es indispensable partir de las experiencias de vida de los profesores con relación al tema. Formarse en interculturalidad implica ante todo una reflexión personal sobre su la relación con el Otro. En esta investigación, este aspecto de concientización sobre la interculturalidad en la historia de vida se logró gracias a la redacción y posterior discusión en grupo de un texto narrativo - una (auto)biografía lingüística-.

A continuación, el espacio de formación debe favorecer el acercamiento de los profesores con elaboraciones conceptuales variadas sobre la educación intercultural. Así lo sugiere Bastos (2015) como recomendación de su estudio y se confirma en el presente trabajo. Además, deben generarse reflexiones y conexiones críticas entre esas teorías y su pertinencia y concreción en el contexto de trabajo de los profesores. Esta reflexión permite no solo esclarecer el posicionamiento de los profesores, sino también tomar acciones didácticas informadas.

Seguidamente, no se debe perder de vista la importancia del trabajo práctico. En otras palabras, es importante que los participantes puedan concretar las ideas teóricas estudiadas en acciones como la elaboración de planes de clase y de materiales para la enseñanza. Los participantes de este estudio sugieren que estas actividades deben alternarse con la lectura y discusión de los textos y no dejarse exclusivamente para la parte final de la formación.

Otro aspecto importante tiene que ver con el rol del formador o líder del grupo de trabajo. Una CP no es el equivalente de un curso universitario con un profesor que posee una experticia y un grupo de estudiantes. Por el contrario, se trata de un grupo que nace, crece, se construye, evoluciona y llega a su fin según los intereses de sus participantes, para quienes, además, no existe ningún tipo de jerarquía. En este sentido, quien lidere el grupo debe participar al mismo título que los demás de las diversas actividades de formación que el grupo establezca dentro de su agenda de trabajo. 
Finalmente, es vital tomar en cuenta algunos aspectos logísticos para el buen funcionamiento de una CP. Por una parte, se sugiere alternar el trabajo en grupo y los espacios de lectura, preparación y reflexión individual. Este estudio mostró que las reuniones de la $\mathrm{CP}$ precedidas de un trabajo de elaboración son más productivas que, por ejemplo, un taller donde un experto presenta un contenido en una sesión. Por otra parte, todo lo que se acuerde horarios, tareas, etc. - dentro de la CP debe hacerse de forma consensuada, solo así podrá garantizarse el compromiso mutuo. El consenso puede lograrse más fácilmente cuando se trata de grupos pequeños, como en el caso de esta investigación, cuya CP contó con ocho personas. Los grupos pequeños permiten, además, que todos tengan la oportunidad de conocerse y de expresarse sobre temas complejos como los que se discuten cuando se habla de interculturalidad, de la relación con el Otro. Finalmente, y no menos importante, la CP debe conservar una memoria de lo que se realizó en su trabajo, es lo que Wenger (1998) llama el repertorio compartido. En el caso del presente estudio, esta consistió en los protocolos de trabajo y en todos los materiales que se leyeron y que se crearon en el marco de la CP.

\section{Conclusiones}

Este estudio confirmó las potencialidades de las CP en la formación permanente del profesorado universitario. Las CP logran la participación en igualdad de condiciones de sus miembros, poniendo a disposición el capital cultural y la experiencia profesional de cada uno de ellos. Además, la participación en la CP contribuye al empoderamiento de los participantes; por ejemplo, dos de las profesoras de francés decidieron comenzar un nuevo proyecto - un nuevo ciclo de formación con otro grupo de profesores-después de culminar este trabajo, con el objetivo de responder a la problemática que presenta el programa, esbozada en la introducción.

Es importante insistir en la temporalidad de esta modalidad de formación. Los datos confirman que, en consonancia con lo señalado por los autores citados, una formación en educación intercultural debe hacerse desde actividades reflexivas que impliquen al sujeto; estas requieren de un tiempo suficiente que no es factible en un curso corto. Si los temas tratados no involucran al sujeto, no pasan por su propia historia de vida, no es posible formarse en algo que por naturaleza exige una reflexividad sobre temas muy complejos que conciernen la relación con el Otro. Así pues, la CP se realizó en un lapso de tiempo amplio que permitió la discusión de los temas relacionados con la interculturalidad, abordándolos desde la experiencia individual y colectiva. Conocer al Otro desde su propia historia de vida y su forma de entender el mundo significó un trabajo alteritario dentro de la misma CP. En otras palabras, la formación en interculturalidad se dio reflexionando y viviendo la alteridad dentro del grupo. Esto fue posible, porque, por un lado, no existía una agenda rígida predeterminada para el trabajo. Por otro lado, realizar el trabajo durante un periodo relativamente largo permitió generar conexiones entre los textos leídos, la propia historia de vida y el actuar como maestros en el aula de clase.

Los hallazgos también reafirman la importancia de una visión renovada y crítica de la interculturalidad en la formación de maestros de lenguas extranjeras y en la enseñanza de lenguas. Estas visiones permiten trascender la idea de la interculturalidad como una competencia reducida a lo que puede ocurrir exclusivamente en un intercambio entre personas originarias de dos países o lenguas diferentes. Por el contrario, una visión renovada y crítica implica que reconozcamos de manera amplia la alteridad en la clase de lenguas. Por supuesto, es importante abordar de manera crítica las culturas extranjeras, pero también es esencial que, desde esta mirada, prestemos atención a quiénes están dentro del aula de clase y a las comunidades del propio país, generalmente soslayadas en las clases de lenguas extranjeras. Esto implica que abandonemos la idea de que nuestros grupos son homogéneos únicamente porque los estudiantes son originarios del mismo país o de la misma región. Se trata, entonces, de reconocer la pluralidad y complejidad de nuestros grupos de trabajo, y de implementar acciones que favorezcan y reconozcan estas 
diversidades en la clase de lenguas. Coincidimos con Dervin en que la interculturalidad debería implicar la co-construcción de las "diversas diversidades" de los individuos que forman parte de un encuentro, en nuestro caso, de los mismos estudiantes y profesores que se encuentran en la clase de lenguas.

\section{Referencias}

Abdallah-Pretceille, M. (1996). Vers une pédagogie interculturelle. Anthropos.

Aguado, T., Gil, I. y Mata, P. (2008). El enfoque intercultural en la formación del profesorado: Dilemas y propuestas. Revista Complutense de Educación, 19(2), 275-292. http://revistas.ucm.es/index.php/ RCED/article/view/RCED0808220275A

Agudelo, J. J. (2007). An intercultural approach for language teaching: Developing critical cultural awareness. Íkala, Revista de Lenguaje y Cultura, 12(1), 185-217. http://aprendeenlinea.udea.edu.co/ revistas/index.php/ikala/article/view/2718

Álvarez, J. A. (2014). Developing the intercultural perspective in foreign language teaching in Colombia: A review of six journals. Language and Intercultural Communication, 14(2), 226-244. http://www. tandfonline.com/doi/abs/10.1080/14708477.2014 .896922

Álvarez, J. A. y Bonilla, S. (2009). Addressing culture in the EFL classroom : A dialogic proposal. Profile, 11(2), 151-170. https://revistas.unal.edu.co/index. php/profile/article/view/11448

Anadón, M. (2006). La recherche dite "qualitative» : de la dynamique de son évolution aux acquis indéniables et aux questionnements présents. Recherches Qualitatives, 26(1), 5-31. http://www.recherche-qualitative.qc.ca/Revue.html

Arismendi, F. (2014). Culture et formation des futurs enseignants de langues : le cas d'un programme de licence dans une université publique en Colombie. Lenguaje, 42(2), 363-387. https://doi.org/https://doi. org/10.25100/lenguaje.v42i2.5864

Arismendi, F. (2016). La competencia plurilingüe y pluricultural en la formación de futuros docentes de lenguas extranjeras en una universidad pública en Colombia. Folios, 44, 109-125. https://doi.org/ https://doi.org/10.17227/01234870.44folios109.125

Bacca, J. E. (2014). Le sens de l'autre: la construction de la signification de l'altérité dans la formation des enseignants avec pour objectif la compétence en communication interculturelle : étude de cas en Colombie [Tesis de doctorado]. Université Nice Sophia Antipolis. https://tel.archives-ouvertes.fr/ tel-01407345

Bardin, L. (2013). L'analyse de contenu (2 ${ }^{\mathrm{a}}$ ed.). Presses universitaires de France.

Barthélémy, F. (2016). Civilisation, culturel, interculturel : objets à haute valeur idéologique dans des projets de transposition complexes en didactique $\mathrm{du}$ FLE. En F. Barthélémy y D. Groux (Eds.), Quarante ans d'interculturel en France. Hommage à Louis Porcher. La revue française d'éducation comparée (pp. 31-55). L'Harmattan.

Bastos, M. (2015). Le professeur interculturel : léducation interculturelle des professeurs de langues dans la formation continue. L'Harmattan.

Bedoya, J. R., Villa, F. L. y Betancourt, M. O. (2018). Creación de una comunidad de práctica para la formación de docentes en la integración de las TiC a los procesos de aprendizaje y enseñanza de lenguas extranjeras. Íkala: Revista de Lenguaje y Cultura, 23(1), 121-139. https://doi.org/10.17533/udea.ikala. v23n01a09

Blanchet, P. y Chardenet, P. (Eds.). (2011). Guide pour la recherche en didactique des langues et des cultures: approches contextualisées. Éditions des archives contemporaines.

Blanchet, P. y Coste, D. (2010). Sur quelques parcours de la notion d' "interculturalité ". Analyses et propositions dans le cadre d'une didactique de la pluralité linguistique et culturelle. En P. Blanchet y D. Coste (Eds.), Regards critiques sur la notion d'interculturalité (pp. 7-27). L'Harmattan.

Bozu, Z. e Imbernon, F. (2009). Creando comunidades de práctica y conocimiento en la Universidad: Una experiencia de trabajo entre las universidades de lengua catalana. Rusc. Universities and Knowledge Society Journal, 6(1), 1-10. https://doi.org/http:// dx.doi.org/10.7238/rusc.v6i1.20

Burns, A. (1999). Collaborative action research for English language teachers. Cambridge University Press.

Byram, M. (1997). Teaching and assessing intercultural communicative competence. Multilingual Matters.

Byram, M., Barrett, M., Ipgrave, J., Jackson, R. y Méndez García, M. del C. (2009). Autobiographie de Rencontres Interculturelles: Contexte, concepts et théories. Conseil de l'Europe, Division des politiques linguistiques. 
http://www.coe.int/t/dg4/autobiography/Source/ AIE_fr/AIE_context_concepts_and_theories_fr.pdf

Cárdenas-Beltrán, M. L., González, A. y Álvarez, J. A. (2010). El desarrollo profesional de los docentes de inglés en ejercicio: Algunas consideraciones conceptuales para Colombia. Folios, 31, 49-68. https:// revistas.pedagogica.edu.co/index.php/RF/article/ view/894/922

Conseil de l'Europe. (2001). Cadre européen commun de référence pour les langues. Apprendre, enseigner, évaluer. Didier.

Conseil de l'Europe. (2018). Cadre européen commun de référence pour les langues: apprendre, enseigner, évaluer. Volume complémentaires avec de nouveaux descripteurs. Conseil de l'Europe.

Coste, D. (2016). Sur quelques aspects de l'approche des cultures et de la compétence interculturelle en didactique des langues. En F. Barthélémy y D. Groux (Eds.), Quarante ans d'interculturel en France. Hommage à Louis Porcher. La revue française d'éducation comparée (pp. 57-79). L'Harmattan.

Cruz, F. (2007). Broadening minds: Exploring intercultural understanding in adult EFL Learners. Colombian Applied Linguistics Journal, 9, 144-173. https://doi. org/https://doi.org/10.14483/22487085.3149

Defays, J.-M. (2018). Enseigner le français langue étrangère et seconde : approche humaniste de la didactique des langues et des cultures. Mardaga.

Delgadillo, I. V. (2016). Représentation linguistique des mots civilisation, culture et interculturel. Quelles constructions identitaires véhiculées par les enseignants et futurs enseignants colombiens de français? [tesis de doctorado]. Université de Nantes. http://archive. bu.univ-nantes.fr/pollux/show.action?id=ecfd $4 \mathrm{~d} 9 \mathrm{~d}$ -fe87-44f3-b06b-1d79782da487

Demorgon, J. (2015). Complexité des cultures et de l'interculturel: contre les pensées uniques (5ème éd.). Anthropos.

Dervin, F. (2011). Impostures interculturelles. L'Harmattan.

Dervin, F. (2016a). Interculturality in education: A theoretical and methodological toolbox. Palgrave Pivot.

Dervin, F. (2016b). L'interculturel en éducation : une notion à retravailler toujours et encore? En F. Barthélémy y D. Groux (Eds.), Quarante ans d'interculturel en France. Hommage à Louis Porcher. La revue française d’éducation comparée (pp. 97-108). L'Harmattan.
Dervin, F. (2017). Compétences interculturelles. Éditions des archives contemporaines.

Erfurt, J. (2016). Le concept de transculturalité : un atout pour le débat sur l'éducation plurilingue. En J. Erfurt y C. Hélot (Eds.), Léducation bilingue en France: politiques linguistiques, modèles et pratiques (pp. 592-609). Lambert-Lucas.

Ferrão, M. (2010). Educación intercultural en América Latina: Distintas concepciones y tensiones actuales. Estudios Pedagógicos, 36(2), 333-342. https://scielo. conicyt.cl/pdf/estped/v36n2/art19.pdf

Galligani, S. (2014). Travail biographique et compétence plurilingue: quels apports en formation des enseignants de langues? En M. Causa, S. Galligani y M. Dudas Vlad (Eds.), Formation et pratiques enseignantes en contextes pluriels (pp. 209-228). Riveneuve éditions.

Gamboa Diaz, P. A. (2019). Second and foreign language pre-service and in-service teacher development to make intercultural dimensions explicit: Reflecting through a comparative proposal between France and Colombia. Voces y Silencios: Revista Latinoamericana de Educación, 10(1), 199-230. https://doi.org/10.18175/vys10.1.2019.14

Gamboa Diaz, P. A., Molinié, M. y Tejada-Sánchez, I. (2019). Accompagner les parcours bilingues/ multilingues/ plurilingues via des dispositifs interculturels. Co-construire la formation des enseignants, un enjeu pour le 21e siècle. Voces y Silencios: Revista Latinoamericana de Educación, 10(1), 3-8. https:// doi.org/10.18175/vys10.1.2019.02

Gómez, C., Álvarez, S. y Gómez, D. (2018). Adjunct instructors: Genesis of a continuing professional development program. Íkala: Revista de Lenguaje y Cultura, 23(3), 545-559. https://doi.org/10.17533/ udea.ikala.v23n03a10

Gómez, L. F. (2013). Enhancing intercultural competence through U. S. multicultural literature in the EFL Classroom. Folios, 1(38), 95-109. http:// revistas.pedagogica.edu.co/index.php/RF/article/ view/2061/1987

Granados-Beltrán, C. (2016). Critical interculturality: A path for pre-service elt teachers. Íkala, Revista de Lenguaje y Cultura, 21(2), 171-187. https://doi. org/10.17533/udea.ikala.v21n02a04

Hernández, O. y Samacá, Y. (2006). A study of EFL students' interpretations of cultural aspects in foreign language learning. Colombian Applied Linguistics 
Journal, 8, 38-52. https://doi.org/https://doi. org/10.14483/22487085.171

Macaire, D. (2007). Didactique des langues et recherche-action. Recherches en Didactique des Langues et Cultures: les Cahiers de l'Acedle, L'association des chercheurs et enseignants en didactique des langues étrangères, 4, 93-119. https://hal.archives-ouvertes. fr/hal-00554863/document

Molinié, M. (2011). La méthode biographique : de l'écoute de l'apprenant de langues à l'herméneutique du sujet plurilingue. En P. Blanchet y P. Chardenet (Eds.), Guide pour la recherche en didactique des langues et des cultures. Approches contextualisées (pp. 144-153). Editions des archives contemporaines.

Molinié, M. (2019). Formation plurilingue et interculturelle des enseignants de langues : historicité, réflexivité, éducation postcoloniale. Voces y Silencios: Revista Latinoamericana de Educación, 10(1), 127143. https://doi.org/10.18175/vys10.1.2019.10

Olaya, A. y Gómez, L. F. (2013). Exploring EfL pre-service teachers' experience with cultural content and intercultural communicative competence at three colombian universities. Profile Issues in Teachers' Professional Development, 15(2), 49-67. http:// www.revistas.unal.edu.co/index.php/profile/article/ view/40168

Peña, B., Tejada-Sánchez, I. y De Mejía, A.-M. (Eds.). (2019). Interculturalidad y formación de profesores: Perspectivas pedagógicas y multilingües. Universidad de los Andes. https://uniandes.edu. co/es/publicacion/libro-interculturalidad-formacion-profesores-perspectiva-pedagogicas-multilingues-beatriz-pena-dix-isabel-tejada-sanchez-anne-marie-truscott-mejia

Perregaux, C. (2002). (Auto)biographies langagières en formation et à l'école: pour une autre compréhension du rapport aux langues. Bulletin VALS-ASLA (Association suisse de linguistique appliquée), 76, 81-94.

Porcher, L. (1986). La civilisation. CLE International.

Porcher, L. y Abdallah-Pretceille, M. (1998). Éthique de la diversité et éducation. Presses Universitaires de France PUF.

Puren, C. (2002). Perspectives actionnelles et perspectives culturelles en didactique des langues-cultures: vers une perspective co-actionnelle-co-culturelle. Les langues modernes, 3, 55-71.
Puren, C. (2011). Modèle complexe de la compétence culturelle (composantes historiques trans-, méta-, inter-, pluri-co-culturelles) : exemples de validation et d'application actuelles. http://www.christianpuren. com/mes-travaux/2011j/

Serna, H. (2016). Integrating the intercultural communicative competence (ICC) in a foreign language program: Faculty considerations upon leaving the haven of native speakership. English Language Teaching, 9(4), 1. https://doi.org/http://dx.doi. org/10.5539/elt.v9n4p1

Serna, H., Quaroni, E., Treuholz, T., Berlincourt, G., Franco, C. y Costa, D. (2016). Comunicación intercultural en el mundo globalizado y la formación multicultural y multilingüe de los profesionales en lenguas modernas. Universidad EAN.

Simon, D.-L. (2014). Ressources biographiques et conscientisation en formation : pour une didactique du plurilinguisme. En M. Causa, S. Calligani y M. Vlad (Eds.), Formation et pratiques enseignantes en contextes pluriels (pp. 191-207). Riveneuve éditions.

Simon, D.-L. (2015). Didactique des langues et éducation plurilingue et interculturelle: l'approche plurielle. Habilitation à diriger des recherches. Université Grenoble Alpes.

Soler, G. (2016). Pour une approche interculturelle de lenseignement/apprentissage du FLE en milieu universitaire colombien: contexte, manques, besoins, propositions [tesis de doctorado]. Université Paul Valéry-Montpellier III. https://tel.archives-ouvertes. fr/tel-01580373/document

Thamin, N. y Simon, D.-L. (2009). Réflexions épistémologiques sur la notion de "biographies langagières". Carnets d'Atelier de Sociolinguistique, 4, 15-33. https://www.u-picardie.fr/LESCLaP/IMG/ pdf/D--L-_Simon_et_N-_Thamin_-_Reflexions_ epistemologiques_sur_la_notion_de_biographies_ langagieres_cle0126fa.pdf

Tremblay, D.-G. (2005). Les communautés de pratique: quels sont les facteurs de succès? Revue internationale sur le travail et la société, 3(2), 52-79. https://oraprdnt.uqtr.uquebec.ca/pls/public/docs/ FWG/GSC/Publication/280/3/8542/1/100535/8/ F1351791388_2005Vol3Num2pp52_79Tremblay. pdf

Tremblay, D.-G. (2006). Les communautés de pratique : lémergence de nouveaux modes d'apprentissage? Communication aux journées de sociologie du 
travail. http://recitsrecettes.org/sites/default/files/ article_cop.pdf

Viafara, J. J. y Ariza, A. (2015). From awareness to cultural agency: EFL Colombian student teachers' travelling abroad experiences. Profile Issues in Teachers' Professional Development, 17(1), 123-141. https://doi.org/https://doi.org/10.15446/profile. v17n1.39499

Walsh, C. (2010). Interculturalidad crítica y educación intercultural. En J. Viaña, L. Tapia y C. Walsh (Eds.), Construyendo interculturalidad crítica (pp. 75-96).
Instituto Internacional de Integración, Convenio Andrés Bello.

Wenger, E. (1998). Communities of Practice: Learning, Meaning, and Identity. Cambridge University Press.

Zarate, G. (1993). Représentations de létranger en didactique des langues. Didier.

\section{Anexos}

\section{Anexo 1. Textos teóricos estudiados en la comunidad de práctica}

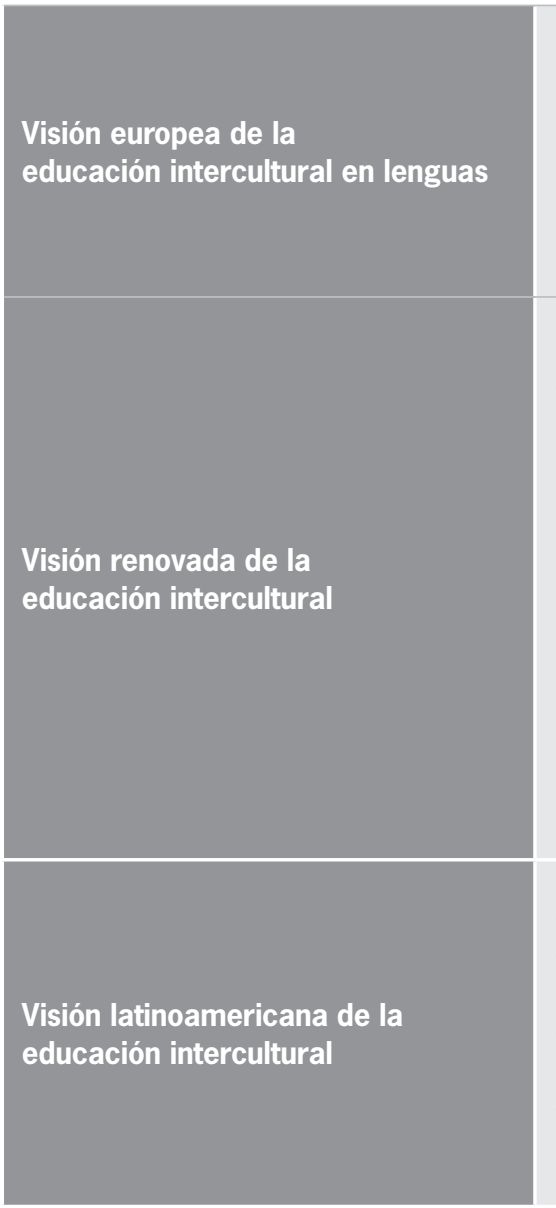

- From Foreign Language Teacher Education to Education for Intercultural Citizenship (Byram, 2008):

- Chapter 4. The intercultural speaker: Acting interculturally or being bicultural. - Chapter 9: Foreign language teaching as political action.

- L'autobiographie des rencontres interculturelles (Byram et al., 2009)

- Pour un humanisme du divers (Abdallah-Pretceille, 2005).

- Interculturality in Education: A Theoretical and Methodological Toolbox (Dervin, 2016).

- Chapter 6. Tools for change: Dynamic and realistic intercultural competences. - Capítulo de conclusión del libro.

- Compétences interculturelles (Dervin, 2017).

- Chapitre 3. Le Graal de la compétence interculturelle.

- Chapitre 9. Développer les compétences interculturelles des enseignants.

- Guía Inter. Introducción a la guía Inter. Módulo 8: Estrategias de enseñanza y aprendizaje (Aguado, 2006).

- Pedagogía intercultural (Aguado, 2003).

- Capítulo 9. Competencias interculturales.

- Interculturalidad crítica y educación intercultural (Walsh, 2010).

- Educación intercultural en América Latina: Distintas concepciones y tensiones actuales (Ferrão, 2010).

- Critical Interculturality. A Path for Pre-service elt Teachers (Granados-Beltrán, 2016).

- Developing the Intercultural Perspective in Foreign Language Teaching in Colombia: A Review of Six Journals (Álvarez, 2014). 


\section{Anexo 2. Guía para la realización del grupo focal}

\section{Objetivos del grupo focal}

- Evaluar el trabajo de formación en interculturalidad llevado a cabo durante el semestre 2018-I.

- Analizar los efectos de la comunidad de práctica en los participantes.

\section{Procedimiento para el desarrollo del grupo focal}

\section{Introducción}

- Agradecer a los participantes por su presencia.

- Explicar brevemente el procedimiento del grupo focal.

\section{Objetivos}

- Presentar a cada uno de los participantes del grupo focal.

- Explicar el rol del moderadory de los participantes.

\section{Procedimiento}

- Explicar que el grupo focal será grabado.

- Aclarar las normas para el buen desarrollo del grupo focal: cada uno se presenta al inicio con el objetivo de identificar las voces en el momento de hacer la transcripción; respetar el turno de cada participante y levantar la mano cuando se desea intervenir para evitar que dos personas hablen al mismo tiempo (con el fin de facilitar la transcripción); respetar el punto de vista de cada participante; intervenir, en la medida de lo posible, en cada una de las preguntas. Verificar que la grabadora funcione bien antes de empezar.

\section{Desarrollo del grupo focal (se}

procede con la guía de preguntas)

\section{Validación de la información: el}

moderador hace una síntesis de las respuestas de los participantes y solicita su confirmación. Si hay imprecisiones, las corrige.

\section{Vl. Agradecimiento a los asistentes}

\section{Guía de preguntas}

1. (Empezar por una pregunta muy general) Cuéntenos, por favor, como vivió la experiencia de participar en esta formación sobre la interculturalidad (incluyendo la escritura del texto narrativo-la biografía lingüística).

2. ¿Cómo contribuyó (o no) el trabajo realizado durante este semestre a su formación continua o desarrollo profesional como formador de futuros maestros de lenguas? En términos concretos, ¿qué se lleva usted de esta formación para su práctica cotidiana como maestro y formador?

3. ¿Considera usted que su visión de la interculturalidad se modificó durante este semestre? ¿Cómo?

4. ¿Cuáles son los aspectos positivos del trabajo realizado durante esta formación?

5. ¿Cuáles son los aspectos por mejorar? ¿Qué le cambiaría usted a este trabajo de formación?

6. Lea por favor la siguiente definición de comunidad de práctica [La definición se presentó en la pantalla del televisor del aula donde se hizo el grupo focal]. Comunidad de práctica: "Un grupo de personas que tienen en común un área de experticia o una práctica profesional, y que se encuentran para intercambiar, compartir y aprender los unos de los otros, cara a cara o virtualmente" (Tremblay, 2005 p. 54). ¿En qué medida considera que el trabajo realizado se corresponde con esta definición? ¿Por qué?

7. Si desea agregar algo más sobre la formación o su contenido, por favor hágalo. 
